\title{
Glypican-1 immunohistochemistry is a novel marker to differentiate epithelioid mesothelioma from lung adenocarcinoma
}

\author{
Vishwa Jeet Amatya ${ }^{1}$, Kei Kushitani ${ }^{1}$, Yuichiro Kai ${ }^{1,2}$, Rui Suzuki ${ }^{1}$, Yoshihiro Miyata ${ }^{2}$, \\ Morihito Okada ${ }^{2}$ and Yukio Takeshima ${ }^{1}$ \\ ${ }^{1}$ Department of Pathology, Hiroshima University Graduate School of Biomedical and Health Sciences, \\ Hiroshima, Japan and ${ }^{2}$ Department of Surgical Oncology, Research Institute for Radiation Biology and \\ Medicine, Hiroshima University, Hiroshima, Japan
}

\begin{abstract}
Histological morphology alone is not sufficient for the pathological diagnosis of malignant mesothelioma. Positive and negative immunohistochemical markers are necessary to differentiate it from lung adenocarcinoma. As calretinin and D2-40, the recognized positive markers of mesothelioma, are expressed in lung adenocarcinoma to some extent, novel markers with high specificity are desirable. In this study, we investigated the applicability of glypican-1 immunohistochemistry to differentiate epithelioid mesothelioma from lung adenocarcinoma. We investigated 82 cases of epithelioid mesothelioma and 97 cases of lung adenocarcinoma for glypican-1 expression by immunohistochemistry using a commercially available antibody. All 82 cases of epithelioid mesothelioma showed glypican-1 expression, most with diffuse and strong reactivity. In contrast, only three cases of lung adenocarcinoma showed focal glypican-1 expression. Glypican-1 expression showed 100 sensitivity, $97 \%$ specificity, and a $98 \%$ accuracy rate to differentiate epithelioid mesothelioma from lung adenocarcinoma. The sensitivity of glypican -1 immunohistochemistry is as high as that of calretinin and D2-40, and its specificity is far better than that of calretinin and D2-40. Therefore, we recommend including glypican -1 immunohistochemistry as a positive marker of epithelioid mesothelioma.
\end{abstract}

Modern Pathology (2018) 31, 809-815; doi:10.1038/modpathol.2017.190; published online 12 January 2018

Malignant mesothelioma is a highly aggressive asbestos-related malignant neoplasm with unfavorable prognosis. The incidence of mesothelioma is still increasing in Japan and Western countries, with an expected peak in the coming decades. ${ }^{1}$ Some of the peripheral lung adenocarcinomas may present as a pseudomesotheliomatous growth pattern and resemble histologically epithelioid mesothelioma. ${ }^{2}$ As the prognosis and treatment protocols of epithelioid mesothelioma and lung adenocarcinoma are different, their differential diagnosis is necessary for the proper management of patients.

Mesothelioma is classified into three major histological subtypes: epithelioid, biphasic, and sarcomatoid mesothelioma. The epithelioid mesotheliomas show various histological growth patterns, ranging from tubulopapillary to solid growth. ${ }^{3}$ Since there is a

Correspondence: Professor Y Takeshima, MD, PhD, Department of Pathology, Hiroshima University Graduate School of Biomedical and Health Sciences, 1-2-3 Kasumi, Minami-ku, Hiroshima 7348551, Japan.

E-mail: ykotake@hiroshima-u.ac.jp

Received 13 September 2017; revised 24 October 2017; accepted 26 October 2017; published online 12 January 2018 strong resemblance between epithelioid mesothelioma and lung adenocarcinoma, immunohistochemical markers are necessary for their accurate diagnosis. The International Mesothelioma Interest Group guideline recommended various immunohistochemical markers of epithelioid mesothelioma and lung adenocarcinoma. Calretinin, D2-40 (podoplanin), WT1, and cytokeratin $5 / 6$ are positive markers for mesothelioma, and CEA, TTF-1, napsin-A, and claudin 4 are positive markers for lung adenocarcinoma. ${ }^{4}$ However, the specificity of calretinin and D2-40 for differential diagnosis of epithelioid mesothelioma is not more than $90 \%$, so caution is required in their interpretation. ${ }^{4}$ We have recently reported two other positive immunohistochemical markers of epithelioid mesothelioma, intelectin-1 and DAB2, identified by gene expression analysis of epithelioid mesothelioma and lung adenocarcinoma. ${ }^{5}$ Although these two positive immunohistochemical markers showed high specificity, their sensitivities were $<80 \%$. Therefore, the search for the novel positive immunohistochemical markers is still desirable. In the present work, we evaluated the ability of glypican-1 expression to 
Table 1 List of Antibodies with their Clone, Commercial Source, and Reaction Conditions

\begin{tabular}{lclcl}
\hline Antibody to & Clone & Source & Dilution & Antigen retrieval \\
\hline Glypican-1 & Polyclonal & Proteintech & $1: 100$ & 60 min, CC1 \\
Calretinin & SP65 & Ventana-Roche & Prediluted & 60 min, CC1 \\
Podoplanin & D2-40 & Nichirei Bioscience & Prediluted & 60 min, CC1 \\
WT1 & 6 F-H2 & Ventana-Roche & $1: 25$ & 60 min, CC1 \\
CEA & COL-1 & Nichirei Bioscience & Prediluted & 60 min, CC1 \\
Claudin 4 & 3E2C1 & Life Technologies & $1: 100$ & 60 min, CC1 \\
TTF-1 & 8G7G3/1 & Dako-Agilent & $1: 25$ & 60 min, CC1 \\
Napsin-A & MRQ-60 & Ventana-Roche & Prediluted & 60 min, CC1
\end{tabular}

Abbreviations: CC1, cell conditioning buffer 1 (Tris-based buffer, pH 8.5 from Ventana-Roche); CEA, carcinoembryonic antigen; TTF-1, thyroid transcription factor-1; WT1, Wilm's tumor 1.

Table 2 Immunohistochemical Result of Epithelioid Mesothelioma and Lung Adenocarcinoma

\begin{tabular}{|c|c|c|c|c|c|c|c|c|c|c|}
\hline \multirow{3}{*}{ Marker } & \multicolumn{5}{|c|}{ Epithelioid mesothelioma (82 cases) } & \multicolumn{5}{|c|}{ Lung adenocarcinoma (97 cases) } \\
\hline & \multirow[b]{2}{*}{ Number of positive cases } & \multicolumn{4}{|c|}{$\begin{array}{l}\text { Immunohistochemical } \\
\text { Score }\end{array}$} & \multirow[b]{2}{*}{ Number of positive cases } & \multicolumn{4}{|c|}{$\begin{array}{c}\text { Immunohistochemical } \\
\text { score }^{\mathrm{a}}\end{array}$} \\
\hline & & 0 & $1+$ & $2+$ & $3+$ & & 0 & $1+$ & $2+$ & $3+$ \\
\hline Glypican-1 & $82(100 \%)$ & 0 & 2 & 24 & 56 & $3(3 \%)$ & 94 & 3 & 0 & 0 \\
\hline Calretinin & $81(99 \%)$ & 1 & 8 & 2 & 71 & $18(19 \%)$ & 79 & 11 & 7 & 0 \\
\hline D2-40 & $79(96 \%)$ & 3 & 7 & 8 & 64 & $7(7 \%)$ & 90 & 5 & 2 & 0 \\
\hline WT1 & $65(79 \%)$ & 17 & 18 & 7 & 40 & $0(0 \%)$ & 97 & 0 & 0 & 0 \\
\hline CEA & $0(0 \%)$ & 82 & 0 & 0 & 0 & $95(98 \%)$ & 2 & 7 & 14 & 74 \\
\hline Claudin 4 & $0(0 \%)$ & 82 & 0 & 0 & 0 & $93(96 \%)$ & 4 & 2 & 11 & 80 \\
\hline TTF-1 & $0(0 \%)$ & 82 & 0 & 0 & 0 & $86(89 \%)$ & 11 & 3 & 10 & 73 \\
\hline Napsin-A & $0(0 \%)$ & 82 & 0 & 0 & 0 & $81(84 \%)$ & 16 & 12 & 2 & 67 \\
\hline
\end{tabular}

Abbreviations: CK, cytokeratin; CEA, carcinoembryonic antigen; TTF-1, thyroid transcription factor-1; WT1, Wilm's tumor 1.

${ }^{\mathrm{a}} 0$ : $0 \%$ or trace; $1+: 1-10 \% ; 2+: 11-50 \% ; 3+:>51 \%$ of tumor cells with immunoreactivity.

differentiate epithelioid mesothelioma from lung adenocarcinoma.

\section{Materials and methods}

\section{Patients and Histologic Samples}

Histological sections of 82 epithelioid mesotheliomas and 97 lung adenocarcinomas were retrieved from the archives of the Department of Pathology, Hiroshima University. No sarcomatoid mesothelioma was included in this study. The patient record files were reviewed for the clinical details and clinical information, such as chest computed tomography findings, to confirm the tumor localization.

Three pathologists (VJA, KK, and YT) reviewed all histological sections. Pathologic diagnosis of all of these cases was confirmed by histologic findings and an additional immunohistochemical marker panel recommended by the 2012 International Mesothelioma Interest Group Guideline ${ }^{6}$ and the 2015 WHO histologic classification. ${ }^{7}$ We collected and used the tissue specimens after the approval of our institutional ethics review committee (Hiroshima University E-974) based on 'The Japanese Ethical Guidelines for Medical and Health Research' enacted by the Japanese Government. Samples used were linked-anonymized archival specimens, and individual consent was exempted for this research.

\section{Immunohistochemical Procedures and Evaluation of Glypican-1 Expression}

Immunohistochemistry was performed using tissue sections prepared from the best representative formalin-fixed, paraffin-embedded blocks of epithelioid mesotheliomas and pulmonary adenocarcinomas. Immunohistochemical staining was performed using the Benchmark GX automated immunohistochemical station (Roche Diagnostics-Ventana, Tokyo, Japan). The antigen retrieval methods and antibodies used in this study are summarized in Table 1. Incubation with the secondary antibody and detection were performed with an ultraView Universal DAB Detection Kit (Roche Diagnostics-Ventana). Cases showing nuclear reactivity for calretinin, WT1, and TTF-1; cytoplasmic reactivity for glypican-1, CEA, and napsin-A; and membranous reactivity for D2-40 and claudin 4 were regarded as positive. This positive immunoreactivity grade was further semiquantified as $0 \%$ for $0 \%$ positive cells or trace staining; $1+$ for up to $10 \%$ of tumor cells showing positive immunostaining; $2+$ for $10-50 \%$ positive tumor cells; and $3+$ for $>50 \%$ 

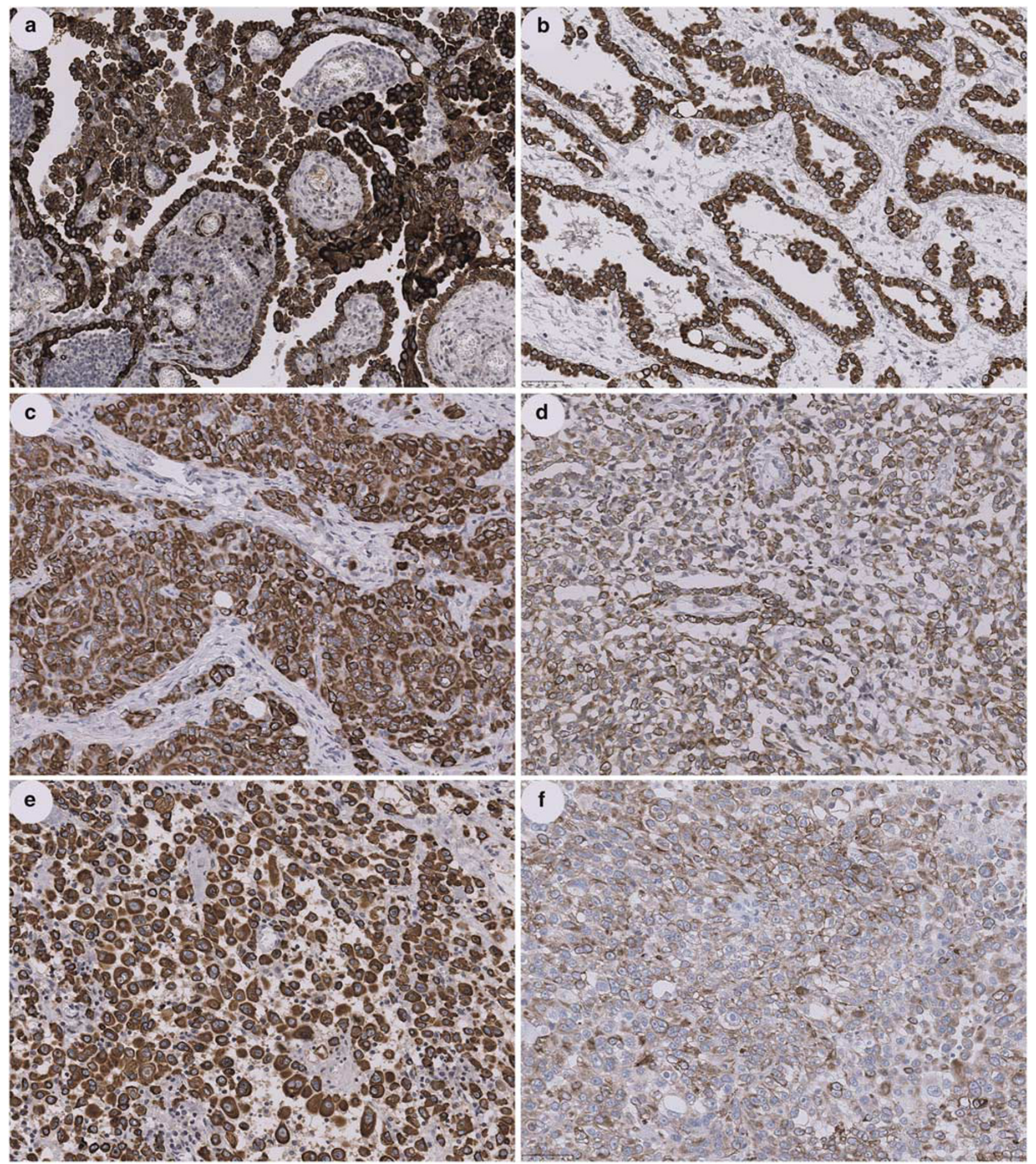

Figure 1 Glypican-1 expression was present in epithelioid mesothelioma of various histological morphologies. (a) Papillary growth; (b) tubular growth; (c) solid-tubular growth; (d) acinar growth; (e) scattered or discohesive growth; (f) solid growth; (g) microcystic growth; and (h) invasive growth into skeletal muscle.

positive tumor cells. Immunohistochemical staining with the ommision of primary antibody is considered as negative control. Bronchial and bronchiolar epithelium for glypican-1, adipose tissue for calretinin, lymphatic vessels for D2-40, and blood vessels for WT1 as internal positive controls were considered as positive controls.

\section{Statistical Analysis}

In this study, the sensitivity was the ability of the marker to correctly identify epithelioid mesothelioma, calculated by the percentage of positive cases (true positive) for the given marker. The specificity was the ability of the immunohistochemical marker 

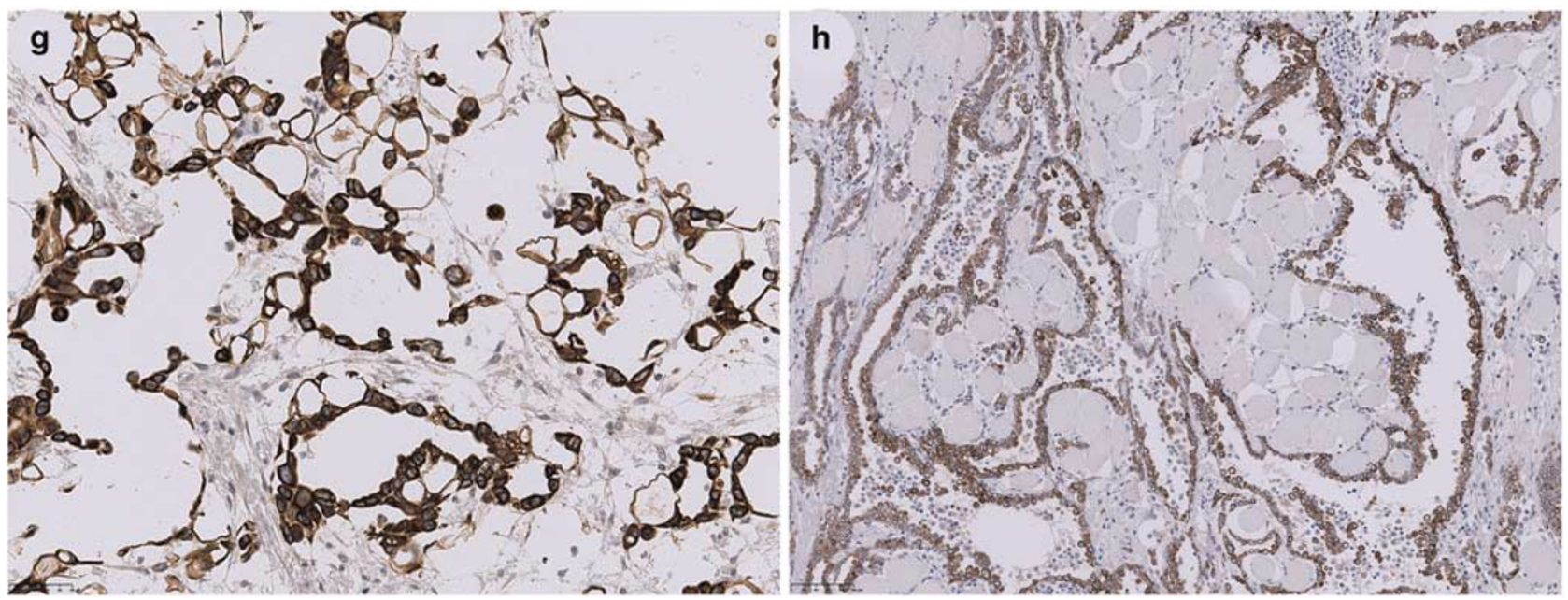

Figure 1 (Continued)

to correctly exclude lung adenocarcinoma, calculated by the percentage of negative cases (true negative) for the given marker. The accuracy rate is expressed as the proportion of cases correctly categorized by the given marker, calculated as a percentage of the sum of true-positive and truenegative cases out of the all cases studied.

\section{Results}

The percentages and immunohistochemical scores of glypican-1 and other immunohistochemical markers in the epithelioid mesothelioma and the lung adenocarcinoma samples are shown in Table 2.

\section{Glypican-1 Expression}

Glypican-1 expression was found in the cytoplasm of tumor cells of epithelioid mesothelioma. It was also observed in basal cells of bronchial and bronchiolar epithelium and normal mesothelial cells, which were considered internal positive controls. Glypican-1 expression was present in all $82(100 \%)$ cases of epithelioid mesotheliomas irrespective of the histological pattern, whether tubulopapillary, cribriform, solid, mucinous, or deciduoid patterns of epithelioid mesothelioma (Figure 1). Among epithelioid mesothelioma cases, 56 cases showed immunohistochemical score $3+, 24$ cases showed score $2+$, and 2 cases showed score 1+. In contrast, glypican-1 expression was not present in 94 of $97(97 \%)$ cases of lung adenocarcinoma (Figure 2). Only three cases of lung adenocarcinoma showed focal and weak glypican-1 expression (immunohistochemical score: 1+).

\section{Calretinin, D2-40, and WT1}

Calretinin, D2-40, and WT1 are positive mesothelial cell markers showing nuclear and cytoplasmic positivity with calretinin, membranous, and cytoplasmic reactivity with D2-40 and nuclear positivity with WT1 in epithelioid mesothelioma. Calretinin was also positive in adipose tissue, D2-40 in lymphatic vessels, and WT1 in blood vessels as positive internal controls, which were considered internal positive controls. Calretinin expression was present in 81 cases (99\%) of epithelioid mesothelioma, 71 of these showing the immunohistochemical score of 3+. However, calretinin expression was also present in 18 of $97(19 \%)$ cases of lung adenocarcinoma, with 7 cases showing the immunohistochemical score of $2+$ and 11 cases showing the immunohistochemical score of 1+. D2-40 expression was present in 79 cases (96\%) of epithelioid mesothelioma, with 64 of these showing the immunohistochemical score of $3+$ and 8 cases showing the immunohistochemical score of 2+. D2-40 expression was also present in 7 of $97(7 \%)$ cases of lung adenocarcinoma, with 2 cases showing the immunohistochemical score of $2+$ and 5 cases showing the immunohistochemical score of 1+. WT1 expression was present in only 65 cases $(79 \%)$ of epithelioid mesothelioma, 40 of these showing the immunohistochemical score of $3+, 7$ cases showing the immunohistochemical score of $2+$, and 18 cases showing the immunohistochemical score of only 1+. WT1 expression was not present in any of 97 cases of lung adenocarcinoma.

\section{CEA, Claudin 4, TTF-1, and Napsin-A}

CEA, claudin 4, TTF-1, and napsin-A expressions were absent from all 82 epithelioid mesotheliomas. CEA and claudin 4 were present in 95 cases (97\%) and 93 cases $(96 \%)$ of lung adenocarcinoma, respectively. The majority of lung adenocarcinomas showed the immunohistochemical score of $3+$ for CEA and claudin 4. TTF-1 and napsin-A were present in 86 (89) and $81(84 \%)$ cases of lung adenocarcinoma with 73 and 67 cases showing the immunohistochemical score of $3+$, respectively. 

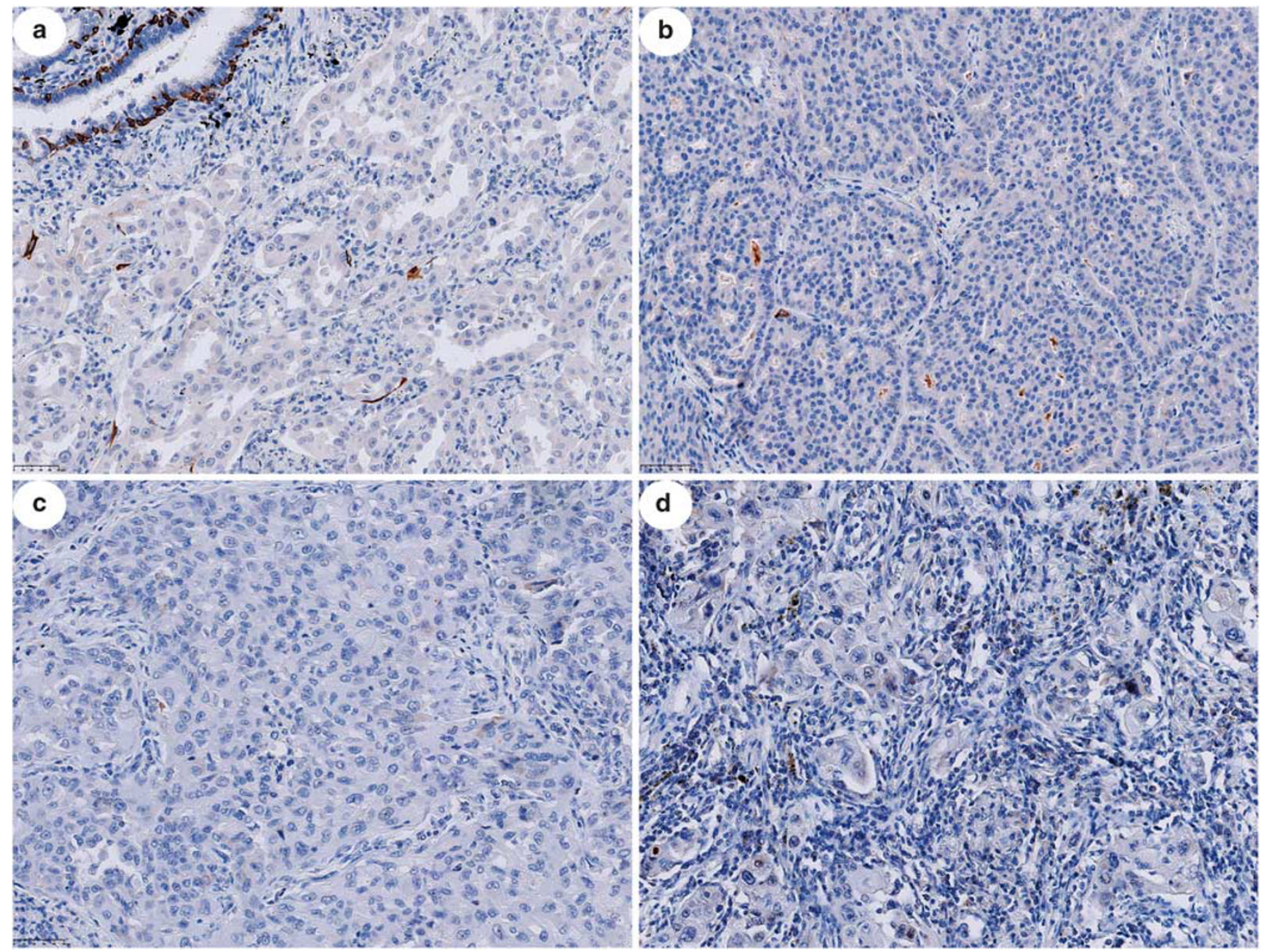

Figure 2 Glypican-1 expression was not detected in lung adenocarcinomas, including papillary (a), acinar (b), solid (c), and discohesive (d). Glypican-1 expression was present in basal cells of the bronchiole (a) and scattered non-neoplastic basal cells (b), as internal positive controls.

Table 3 Sensitivity, specificity, and accuracy rate of immunohistochmical markers for differentiation of epithelioid mesothelioma from lung adenocarcinoma

\begin{tabular}{lccc}
$\begin{array}{l}\text { Immunohistochemical } \\
\text { markers }\end{array}$ & $\begin{array}{c}\text { Sensitivity } \\
(\%)\end{array}$ & $\begin{array}{c}\text { Specificity } \\
(\%)\end{array}$ & $\begin{array}{c}\text { Accuracy rate } \\
(\%)\end{array}$ \\
\hline Glypican-1 (+) & 100 & 97 & 98 \\
Calretinin (+) & 99 & 81 & 89 \\
D2-40 (+) & 97 & 93 & 94 \\
WT1 (+) & 86 & 100 & 91 \\
CEA (-) & 100 & 98 & 99 \\
Claudin 4 (-) & 100 & 96 & 98 \\
TTF-1 (-) & 100 & 89 & 94 \\
Napsin-A (-) & 100 & 84 & 91 \\
\hline
\end{tabular}

Abbreviations: CEA, carcinoembryonic antigen; TTF-1, thyroid transcription factor-1; WT1, Wilm’s tumor 1.

\section{Sensitivity, Specificity, and the Accuracy Rate of Immunohistochemical Markers}

Table 3 shows the sensitivities, specificities, and accuracy rates of the immunohistochemical markers in differentiating epithelioid mesothelioma from lung adenocarcinoma. Positive expression of glypican-1 showed a sensitivity of $100 \%$, specificity of $97 \%$, and an accuracy of $98 \%$. Among the positive mesothelioma markers, positive expression of WT1 showed the highest specificity of $100 \%$, but its sensitivity was limited to $79 \%$. The sensitivities of positive expression of calretinin and D2-40 were high, at 99 and $96 \%$, respectively, but the specificities were limited to $81 \%$ and $93 \%$. Negative expression of CEA, claudin 4, TTF-1, and napsin-A, showed sensitivities of $100 \%$ and specificities of $98 \%, 96 \%, 89 \%$, and $84 \%$, respectively.

\section{Discussion}

The International Mesothelioma Interest Group guideline has recommended a panel of positive markers of epithelioid mesothelioma, but precaution is needed when interpreting calretinin and D2-40 due to for their low specificity and WT1 for its low sensitivity. To improve the accuracy of the diagnosis of epithelioid mesothelioma, we recently reported two positive 
immunohistochemical markers of epithelioid mesothelioma, intelectin-1 and DAB2, identified by differential gene expression analysis of epithelioid mesothelioma and lung adenocarcinoma. ${ }^{5}$ Although these two new immunohistochemical markers showed high specificity, their sensitivities were not high enough, leading us to search for additional immunohistochemical markers.

Glypican-1 is one of the six glycosylphosphatidylinositol-anchored, cell-surface heparan sulfate proteoglycans that play vital roles in growth factor signaling. ${ }^{8}$ Among the six different glypicans, only glypican-3 expression has been studied in mesothelioma, and it is largely silenced due to hypermethylation of its promoter region. ${ }^{9}$ There is no available study of glypican-1 in malignant mesothelioma. Increased expression of glypican-1 is reported in breast cancer, ${ }^{10}$ pancreatic cancer, ${ }^{11}$ esophageal squamous cell carcinoma, ${ }^{12}$ and gliomas. ${ }^{13}$ However, these reports did not have detailed expression patterns. In the Human Protein Atlas, which shows the expression and localization of human proteins across tissues and organs, based on deep sequencing of RNA (RNA-seq) from 37 major different normal tissue types and immunohistochemistry on tissue microarrays containing 44 different tissue types, ${ }^{14}$ most of the human malignancies showed decreased expression of glypican-1 (https://www.proteinatlas. org/search/GPC1). Mesothelioma and mesothelial tissues are not included in this database. In the present study, we first investigated glypican-1 expression in epithelioid mesothelioma by immunohistochemistry and were surprised to find its expression in epithelioid mesotheliomas irrespective of their histological heterogeneity. It was also expressed in non-neoplastic mesothelial cells to some extent. These findings prompted us to study the possibility of glypican-1 immunohistochemistry as a novel positive mesothelioma marker. We still do not know the detailed reason of glypican-1 expression in mesothelial cells, but we suppose that the expression of glypican-1 in the non-neoplastic mesothelial cell can be considered valuable for its application as a positive mesothelioma marker. In peripheral lung tissue, glypican-1 was expressed in basal cells of bronchial tissue, and we utilized this as an internal positive control in lung adenocarcinoma cases. The biology of glypican-1 expression was not fully understand except its probable role in development of the various tissue. Glypican-1 expression was related to promote angiogenesis, metastasis, cell division, and perineural invasion of pancreatic cancer cells ${ }^{15}$ and has been reported as possible prognostic marker of pancreatic cancer. ${ }^{16}$ Recently, Melo et al. ${ }^{17}$ reported the presence of glypican-1 in exosomes in the circulation of pancreatic cancer patients, suggesting the possibility of glypican-1 as a candidate for detection of exosomes for genetic and proteomic analysis in the early pancreatic cancer. Harada et al. ${ }^{18}$ reported the increased expression of glypican-1 in esophageal squamous cell carcinoma and anti-glypican-1 mAb as a therapeutic strategy to decrease tumor growth in patients with glypican-1positive esophageal squamous cell carcinoma. Further biological and functional analysis are needed to draw conclusions about its importance in malignant mesothelioma. In this study, we did not yet included sarcomatoid mesothelioma or various other human carcinomas and sarcoma. However, we are planning to study the glypican-1 expression pattern in various normal tissues and cancerous tissues of various organs and its functional significance in mesothelioma in the near future.

In conclusion, glypican-1 is a novel positive marker of epithelioid mesothelioma with better sensitivity and specificity than calretinin, D2-40, and WT1 for differentiation from lung adenocarcinoma. Further analysis is necessary to fully understand glypican-1 expression in mesothelioma, including sarcomatoid mesothelioma and other malignancies. We recommend including glypican-1 immunohistochemistry as a positive immunohistochemical marker for differential diagnosis of epithelioid mesothelioma from lung adenocarcinoma.

\section{Acknowledgments}

We thank Yukari Go and Tasuya Nakagawa of the Technical Center in Hiroshima University for excellent technical assistance, and Naomi Fukuhara for administrative support. This work was supported by JSPS KAKENHI Grant Number 17K08742.

\section{Disclosure/conflict of interest}

The authors declare no conflict of interest.

\section{References}

1 Delgermaa V, Takahashi K, Park EK, et al. Global mesothelioma deaths reported to the World Health Organization between 1994 and 2008. Bull World Health Organ 2011;89:716-724.

2 Attanoos RL, Gibbs AR. 'Pseudomesotheliomatous' carcinomas of the pleura: a 10-year analysis of cases from the Environmental Lung Disease Research Group, Cardiff. Histopathology 2003;43:444-452.

3 Galateau-Salle F, Churg A, Roggli V, Travis WD. World Health Organization Committee for Tumors of the P. The 2015 World Health Organization Classification of Tumors of the Pleura: advances since the 2004 classification. J Thorac Oncol 2016;11:142-154.

4 Husain AN, Colby TV, Ordonez NG, et al. Guidelines for pathologic diagnosis of malignant mesothelioma: 2017 Update of the Consensus Statement From the International Mesothelioma Interest Group. Arch Pathol Lab Med 2017; doi: 10.5858/arpa.2017-0124RA.

5 Kuraoka M, Amatya VJ, Kushitani K, et al. Identification of DAB2 and intelectin-1 as novel positive immunohistochemical markers of epithelioid mesothelioma by transcriptome microarray analysis for its 
differentiation from pulmonary adenocarcinoma. Am J Surg Pathol 2017;41:1045-1052.

6 Husain AN, Colby T, Ordonez N, et al. Guidelines for pathologic diagnosis of malignant mesothelioma: 2012 Update of the Consensus Statement from the International Mesothelioma Interest Group. Arch Pathol Lab Med 2013;137:647-667.

7 Travis WD, Brambilla E, Nicholson AG, et al. The 2015 World Health Organization Classification of Lung Tumors: impact of genetic, clinical and radiologic advances since the 2004 classification. J Thorac Oncol 2015;10:1243-1260.

8 Lamoureux F, Baud'huin M, Duplomb L, Heymann D, Redini F.. Proteoglycans: key partners in bone cell biology. BioEssays 2007;29:758-771.

9 Murthy SS, Shen T, De Rienzo A, et al. Expression of GPC3, an X-linked recessive overgrowth gene, is silenced in malignant mesothelioma. Oncogene 2000;19: 410-416.

10 Matsuda K, Maruyama H, Guo F, et al. Glypican-1 is overexpressed in human breast cancer and modulates the mitogenic effects of multiple heparin-binding growth factors in breast cancer cells. Cancer Res 2001;61:5562-5569.

11 Kleeff J, Ishiwata T, Kumbasar A, et al. The cell-surface heparan sulfate proteoglycan glypican-1 regulates growth factor action in pancreatic carcinoma cells and is overexpressed in human pancreatic cancer. J Clin Invest 1998;102:1662-1673.

12 Hara H, Takahashi T, Serada S, et al. Overexpression of glypican-1 implicates poor prognosis and their chemoresistance in oesophageal squamous cell carcinoma. Br J Cancer 2016;115:66-75.

$13 \mathrm{Su}$ G, Meyer K, Nandini CD, et al. Glypican-1 is frequently overexpressed in human gliomas and enhances FGF-2 signaling in glioma cells. Am J Pathol 2006;168:2014-2026.

14 Uhlen M, Zhang C, Lee S, et al. A pathology atlas of the human cancer transcriptome. Science 2017;357:357.

15 Aikawa T, Whipple CA, Lopez ME, et al. Glypican-1 modulates the angiogenic and metastatic potential of human and mouse cancer cells. J Clin Invest 2008;118: 89-99.

16 Duan L, Hu XQ, Feng DY, et al. GPC-1 may serve as a predictor of perineural invasion and a prognosticator of survival in pancreatic cancer. Asian J Surg 2013;36: 7-12.

17 Melo SA, Luecke LB, Kahlert C, et al. Glypican-1 identifies cancer exosomes and detects early pancreatic cancer. Nature 2015;523:177-182.

18 Harada E, Serada S, Fujimoto M, et al. Glypican-1 targeted antibody-based therapy induces preclinical antitumor activity against esophageal squamous cell carcinoma. Oncotarget 2017;8:24741-24752. 